Service social

\title{
Intervention sociale en milieu syndical : une expérience d'approche intégrée
}

\section{Michelle Desrosiers}

Volume 36, numéro 2-3, 1987

Approches intégrées

URI : https://id.erudit.org/iderudit/706370ar

DOI : https://doi.org/10.7202/706370ar

Aller au sommaire du numéro

Éditeur(s)

École de service social de l'Université Laval

ISSN

1708-1734 (numérique)

Découvrir la revue

Citer cet article

Desrosiers, M. (1987). Intervention sociale en milieu syndical : une expérience d'approche intégrée. Service social, 36(2-3), 397-412.

https://doi.org/10.7202/706370ar
Résumé de l'article

Il s'agit d'une réflexion sur une expérience portant sur la gestion du stress au travail et vécue avec des conseillers syndicaux à Québec.

Après avoir situé la problématique et l'orientation théorique d'intervention, l'auteure décrit l'expérience et en précise les limites, les résultats et les données d'évaluation 


\section{Intervention sociale en milieu syndical : une expérience d'approche intégrée *}

\section{Michelle Desrosiers}

Depuis une quinzaine d'années, la gestion des services sociaux a été axée sur la rationalisation et la rentabilité de la production des services. Ce tournant administratif a provoqué une période d'ajustements entre la qualité et la quantité des services sociaux publics et a modifié l'accessibilité à ces même services. Dans ce contexte où la distribution des services est remaniée presque constamment, quelle est notre marge de manœuvre, comme intervenantes ? ${ }^{1}$ Comment pouvons-nous tenir compte des nouvelles conditions de travail imposées par cette crise? Comment pouvons-nous développer des plans d'intervention permettant de répondre aux nouveaux besoins engendrés par cette même situation? Comment profiter de nos habiletés pour développer des réponses novatrices et ponctuelles?

Les pages qui suivent offrent une réflexion liée à l'analyse-synthèse d'une expérience portant sur la gestion du stress au travail et vécue avec des conseillers syndicaux d'une importante centrale syndicale à Québec. Les principaux points de l'approche structurelle que nous qualifions d'approche intégrée sont d'abord rappelés sommairement, ainsi que la façon dont nous les avons orchestrés avec d'autres approches généralement utilisées en milieu syndical. La conclusion constituera, nous l'espérons, le point de départ vers de nouvelles expériences à tenter, à analyser et à vivre.

\section{La problématique}

Nous vivons actuellement un contexte de réorganisation à plusieurs niveaux. La profession de service social n'a pas été et n'est pas à l'abri des effets secondaires créés par la complexité de cette situation. La 
légitimation des coupures de postes et de salaires, la réduction des services à la population, la perte des droits acquis et la diminution d'autonomie sociale, économique et politique sont des réalités qui ont touché l'ensemble des travailleurs et des travailleuses au Québec.

Le personnel des secteurs publics de santé et de services sociaux étant composé majoritairement de femmes syndiquées œuvrant auprès d'une clientèle souvent syndiquée aussi, les conditions de travail de ces personnes nous intéressent beaucoup. De plus, considérant que leur sort est régi par des conventions collectives signées par le patronat et le syndicat, que la marge de manœuvre de pouvoir des travailleuses(eurs) se retrouve dans leur allégeance syndicale, que les conseillers syndicaux ${ }^{2}$ sont les principaux agents de gestion du pouvoir des travailleurs et travailleuses, nous les avons retenus comme les cibles de notre intervention.

Nous formulons l'hypothèse que, dans le contexte actuel, les conseillers syndicaux puissent constituer un axe autour duquel nous espérons avoir vécu une expérience de changement personnel et social dont les effets synergiques ont bénéficié à l'ensemble des travailleurs ainsi que dans les milieux de travail où ils ont des responsabilités professionnelles.

Afin de cautionner notre action en milieu syndical, nous avons retenu le stress au travail comme thème central de l'expérience d'intervention. Nous avions aussi la préoccupation de structurer cette expérience de façon à ce qu'elle profite personnellement aux participants ainsi qu'aux membres de leur famille, pour déborder enfin dans leur propre milieu de travail et chez ceux dont ils ont la responsabilité syndicale.

Pour concrétiser la démarche, nous avons développé un processus et organisé un contenu, en puisant conjointement dans les théories du service social des groupes ${ }^{3}$ et de l'approche structurelle. ${ }^{4}$

\section{Quelques orientations théoriques d'intervention sur le stress au travail}

On retrouve peu d'expériences d'intervention chez les auteurs qui se sont intéressés au concept du stress au travail. Suite à nos lectures et à nos démarches auprès de firmes de consultation et de recherche en gestion, trois tendances se dégagent et nous croyons pertinent de les présenter sommairement. 


\section{Première orientation}

L'intervention est ici centrée sur le changement de l'individu. À partir du point de vue que chaque personne n'a pas le même seuil de tolérance face au stress au travail, on propose différentes solutions individuelles susceptibles de l'aider à s'adapter ou à mieux gérer son adaptabilité au stress.

On suppose donc que la personne est seule responsable du maintien de son autonomie sans remettre en question l'environnement. Cette vision a donné naissance à la mise sur pied, dans les milieux de travail, de sessions de relaxation, d'exercices physiques, de méditation. ${ }^{5}$ La figure $I$ indique la direction du changement.

\section{FIGURE I}

\section{Cible d'intervention : la dynamique personnelle}

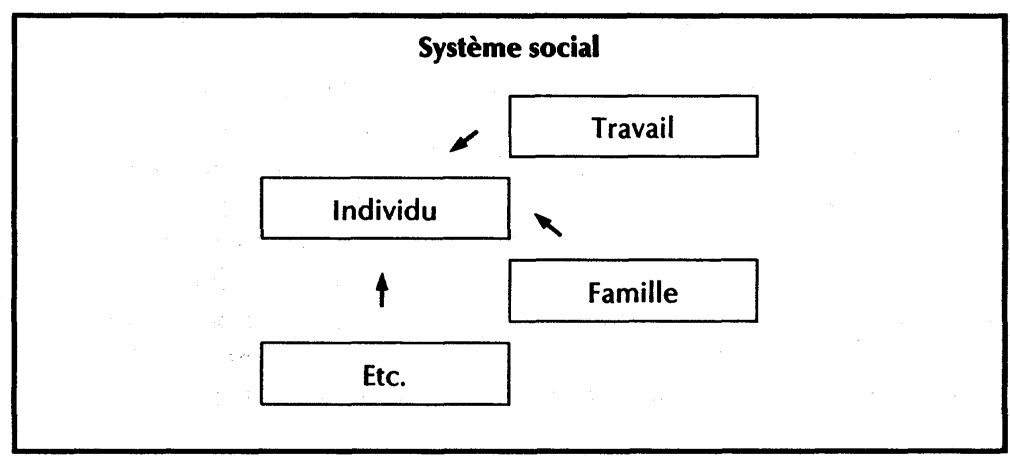

Bien que les bienfaits de ce type d'approche soient réels, nous croyons néanmoins qu'agir sur l'individu n'est que le point de départ d'un changement qui peut éventuellement déteindre sur d'autres systèmes. Le stress de la travailleuse n'est pas séparé des autres dimensions de sa vie.

\section{Deuxième orientation}

Contrairement au premier, un deuxième modèle oriente l'action vers le changement de l'environnement au travail. 
Plusieurs facteurs contribuent à l'augmentation du stress au travail : la croissance d'une compagnie, la production à plus large échelle, l'application de nouvelles formes et normes de travail, la centralisation des décisions, le roulement de personnel, etc. Ici, le bouc émissaire, c'est l'environnement. ${ }^{6}$

\section{FIGURE 2}

\section{Cible d'intervention : la dynamique du milieu de travail}

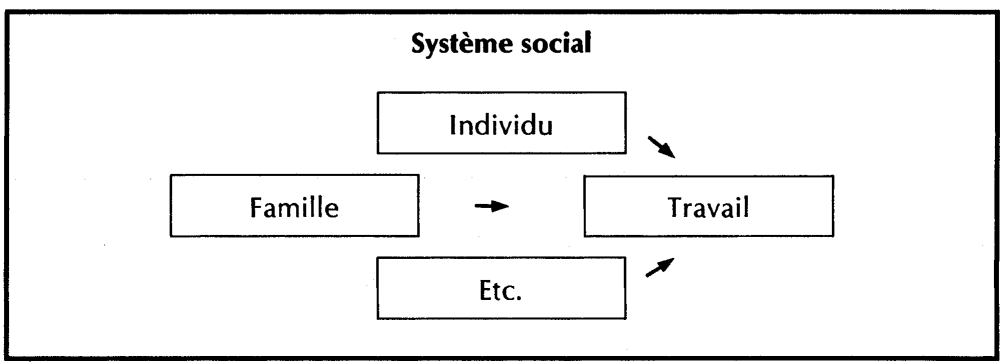

Des stratégies telles les vacances planifiées, l'analyse institutionnelle, les programmes de formation, ont contribué au développement du concept de l'organisation du travail et les sciences du "management " en ont poursuivi le développement.

À notre avis, cette seconde tendance va plus loin que la première : elle n'est pas basée uniquement sur le manque de capacité de l'individu à s'adapter à diverses situations, mais elle soupçonne l'environnement d'être la principale cause du stress au travail. L'addition des forces de ces deux courants a contribué au développement du troisième.

\section{Troisième orientation}

En effet, ce courant vise à la fois le développement de l'individu et de l'environnement. ${ }^{7} \mathrm{C}$ 'est par un travail de synergie que se maximisent les effets positifs d'adaptation au stress en général, et donc aussi au travail. Un équilibre satisfaisant se vit quand la motivation d'une personne pour son travail s'ajuste aux motivations du milieu de travail pour la qualité de vie de ses membres. Les autres systèmes de notre vie personnelle, familiale, économique, etc. viennent nuancer le vécu pris dans son ensemble. L'action se veut donc plus écologique. Voici le schéma illustrant ce troisième courant. 


\section{FIGURE 3}

\section{Cible d'intervention :} la dynamique de la personne/l'environnement

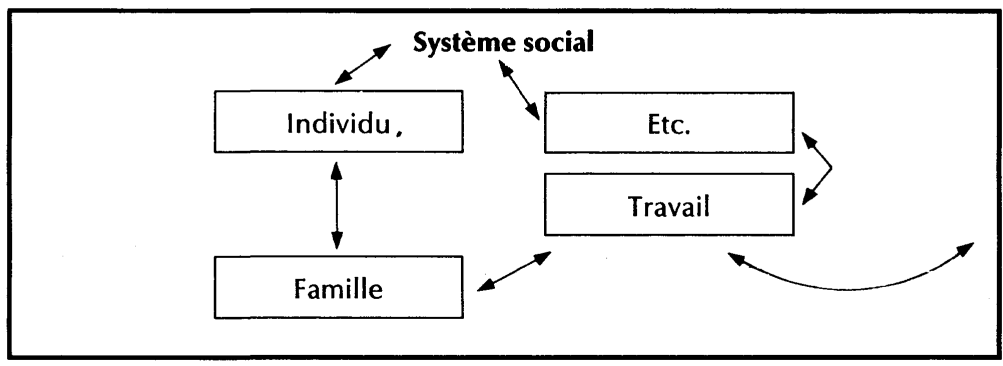

Les trois orientations énoncées ont leurs forces et leurs faiblesses, mais aucune d'elles ne fait l'analyse politique du stress au travail. À l'aide de cette dernière analyse, nous avons donc développé une quatrième orientation, qui tient compte de l'aspect politique du stress, et nous l'avons encadrée par l'approche structurelle.

\section{L’approche structurelle}

Le modèle structurel tel que défini par Moreau ${ }^{8} \mathrm{a}$ comme premier objectif "la réduction du rapport de pouvoir inégalitaire entre la travailleuse sociale et la cliente en vue de favoriser le développement d'une relation dialogique entre elles, c'est-à-dire d'une relation caractérisée par un échange horizontal plutôt que vertical ». ${ }^{9}$

Il s'agit d'une tentative de réduction des distances sociales entre la consultante et la consultée, doublée du souci de démystification du savoir dans leur relation. Par le filtre des valeurs, des perceptions et des interprétations respectives qu'elles mettent en commun, elles établissent et définissent ensemble le partage des possibilités et des limites inhérentes à chacune dans le contexte qui leur est spécifique.

"Le second objectif de l'approche structurelle consiste à comprendre et à tenter de résoudre les problèmes sociaux dans leur contexte social, politique et économique. Le postulat de base, ici, est que les conditions matérielles objectives engendrées par le patriarcat et le mode de production capitaliste déterminent avant tout la façon dont les hommes et les femmes, selon leur classe sociale, pensent, agissent et se sentent. " ${ }^{10}$ 
Cela revient à affirmer qu'il est très important de tenir compte du sexe et des conditions sociale, économique et psychologique dans lesquelles la personne pose son existence, avec les effets provoqués par chacune de ces conditions. Il importe donc : " [...] de toujours comprendre les effets des conditions matérielles - de travail et de vie - sur les comportements personnels et interpersonnels et d'autre part, dans la mesure du possible, de toujours intervenir pour changer ces conditions avant d'intervenir aux niveaux personnels ou interpersonnels ". " L'auteur de cette approche a comme premier souci « d'aider les gens à développer une "praxis" sociale, c'est-à-dire à en arriver à une réflexion critique sur les problèmes personnels/politiques vécus, suivi d'une action aux niveaux personnel et/ou collectif ". 12

Cela soulève des questions dans la pratique sociale : à qui profite la situation ? quel est le prix du changement ? qu'est-ce que je suis capable de payer? Nous tenterons de répondre à ces interrogations dans les pages suivantes par la description de l'utilisation de cette approche dans une expérience en milieu syndical.

\section{Application de l'approche structurelle au milieu syndical}

Par un effort alliant l'analyse de la problématique, la troisième orientation de résolution de problèmes de stress en milieu de travail et l'application du service social des groupes, nous avons tenté une expérience structurée et intégrée de gestion du stress en milieu syndical.

Quant à leur conception du changement social, nous avons constaté beaucoup de similitude entre l'idéologie de l'approche structurelle et celle de la centrale syndicale où s'est vécue l'intervention. Le tableau 1 en témoigne.

De plus, nous avions la prétention d'expérimenter l'approche structurelle d'une façon intégrée, soit avec une structure d'action visant les différents niveaux de changement : changement personnel, développement des membres, changement social, efficacité et humanisation des services. ${ }^{13}$ Le second tableau vient appuyer notre démarche. La priorité de notre action se situant au niveau du changement social, le second tableau réunit les principaux éléments de cette pratique. 


\section{TABLEAU 1}

\section{Objectifs d'analyse et d'action de la centrale syndicale et de l'approche structurelle}

\begin{tabular}{|c|c|}
\hline $\begin{array}{l}\text { Objectifs généraux } \\
\text { de la centrale syndicale }\end{array}$ & $\begin{array}{l}\text { Objectifs généraux } \\
\text { de l'approche structurelle }\end{array}$ \\
\hline $\begin{array}{l}\text { - Abolition des inégalités sociales, } \\
\text { surtout celles liées au travail }\end{array}$ & $\begin{array}{l}\text { - Réduction, voire abolition des iné- } \\
\text { galités sociales, politiques, éco- } \\
\text { nomiques }\end{array}$ \\
\hline $\begin{array}{l}\text { - Amélioration de la qualité de vie } \\
\text { au travail }\end{array}$ & $\begin{array}{l}\text { - Démystification de la création de } \\
\text { bouc émissaire qui sert souvent à } \\
\text { légitimer l'inégalité }\end{array}$ \\
\hline $\begin{array}{l}\text { - Démocratisation du travail et ges- } \\
\text { tion, par la recherche de nouvelles } \\
\text { approches, de nouvelles relations } \\
\text { de travail }\end{array}$ & $\begin{array}{l}\text { - Réappropriation de nos capacités } \\
\text { d'agir comme personne et comme } \\
\text { collectivité }\end{array}$ \\
\hline $\begin{array}{l}\text { - Création de nouvelles valeurs face } \\
\text { au travailleur, vu en interrelation } \\
\text { avec d'autres systèmes }\end{array}$ & $\begin{array}{l}\text { - Conscientisation en rapport avec } \\
\text { ce qui précède pour guider des } \\
\text { prises de décisions et des actions } \\
\text { réalistes }\end{array}$ \\
\hline
\end{tabular}

\section{TABLEAU 2}

\section{Principes d'utilisation du groupe visant le changement social}

1. Les membres doivent être capables de fonctionner aussi bien individuellement que collectivement pour constituer un groupe dont la force unifie l'action.

2. Le groupe doit supporter ses membres contre les résistances externes afin de changer ce qui apparaît généralement comme une réaction.

3. Le groupe doit se donner des récompenses afin d'encourager et supporter les changements positifs et éviter les déviances qui pourraient provoquer des sanctions sociales négatives.

4. Le groupe doit avoir accès à des stratégies personnelles, de groupe et aussi de sous-groupe.

5. Les ressources financières et les informations nécessaires doivent être disponibles afin que le groupe puisse prendre les actions pour atteindre ses objectifs.

6. Généralement, il est aussi essentiel, pour influencer le changement du système, d'avoir accès aux différents moyens de communication et d'information afin de structurer le support et la reconnaissance de l'action. 


\section{Description de l'expérience}

\section{But de l'intervention}

L'expérience visait une réappropriation du pouvoir personnel et collectif sur le changement face aux insatisfactions identifiées par les participants et concernant l'impact du stress dans leur vie personnelle et professionnelle. En privilégiant le milieu syndical comme cible d'intervention, nous voulions expérimenter une action intégrée de changement dans un lieu sortant des sentiers battus, et où une intervention était justifiée. Nous voulions aussi contribuer au développement de l'application de l'approche structurelle et au développement de nouvelles avenues d'intervention en travail social.

Sachant que bien peu de travailleuses sociales ont ou ont eu à intervenir en milieu syndical, nous voulions :

- sensibiliser les intervenantes sociales (syndiquées ou non) à utiliser davantage le pouvoir des ressources syndicales de leur propre milieu de travail et les motiver à sensibiliser leur clientèle à reproduire cette action,

- éveiller les intervenantes à la recherche de nouvelles avenues ponctuelles où le travail social comblerait des réels besoins,

- expérimenter une action structurée et intégrée de changement avec un groupe de travailleurs en situation de pouvoir, pour maximiser la transmission du processus de changement vécu.

\section{Composition du groupe}

Il était formé de huit permanents d'une importante centrale syndicale de Québec, dont l'âge variait entre trente-trois et cinquante ans. Le groupe peut être qualifié d'homogène, la seule femme en était l'intervenante, soit l'auteure du présent article.

Les participants ont été approchés individuellement par le secrétaire de la centrale syndicale, suite à une étude du projet que nous lui avions proposé. Sur une possibilité de dix-neuf personnes, huit conseillers syndicaux se sont présentés à la rencontre d'information et ont vécu le processus de l'intervention du début à la fin. Tous étaient mariés et devaient donc composer avec les réalités de leur vie professionnelle, familiale, sociale et personnelle. De fait, la majorité des personnes étant "permanents ou conseillers" syndicaux sont des hommes. Notons que le personnel clérical, majoritairement féminin, n'a pas été invité à participer au groupe. N'est-ce pas une illustration de la division sexiste du travail ? 14 
Les travailleurs et travailleuses étant la force de travail de toute organisation, les permanents syndicaux s'avéraient, dans le cadre de cette démarche, le groupe par excellence pour expérimenter une intervention hors du réseau traditionnel des services sociaux et tenter d'apporter des réponses aux nouveaux besoins auxquels la pratique du travail social pourrait répondre.

Nous avons donc vécu, entre octobre et décembre 1982, une série de neuf rencontres structurées d'une durée variant entre cinq et sept heures.

\section{Processus de l'intervention}

L'intervenante avait la pleine responsabilité du processus et les participants étaient responsables du contenu et de leur investissement personnel dans la démarche. Celle-là s'est de plus retrouvée dans une situation de leader d'un groupe d'hommes très articulés et visant l'efficience dans l'intervention. Les paragraphes qui suivent décrivent les principales tâches sur lesquelles se sont penchés les participants.

Identification des réalités individuelles et privées. Avec une série de grilles construites par l'intervenante (dont la synthèse est reporté à l'annexe 1) ou inspirées de différents auteurs ${ }^{15}$ qui se sont intéressés au stress au travail, les participants ont identifié leurs malaises physiques, émotifs et psychologiques.

À titre d'exemples: fatigue, douleurs dans la poitrine, migraines, constipations, sentiment d'être forcé de trouver des solutions à tout, sentiment d'impuissance et d'écrasement, déchirement entre le temps passé au travail, celui vécu avec la famille, et le temps qui reste pour soi.

Démystification et collectivisation des réalités privées. Les réalités privées identifiées et liées à l'état de santé, ont débouché sur une mise en commun des informations. Cette étape a permis, à travers des échanges verbaux et structurés entre les membres, de démystifier "le privé " et de prendre conscience de la dimension collective de leurs malaises.

En utilisant d'autres grilles de travail, ${ }^{16}$ les participants ont précisé le contexte dans lequel ces malaises se manifestaient. Ils ont aussi mesuré leur vulnérabilité, identifié leur type de personnalité face au stress, l'état de leur condition physique ${ }^{17}$ et leur degré de danger face à l'épuisement professionnel. ${ }^{18}$ La méthode suivie lors de ces exercices fut d'abord la prise de conscience des données privées, pour ensuite les démystifier et les décoder par une mise en commun. 
Définition des réalités liées aux conditions de travail. À cette étape, les membres du groupe ont identifié les frustrations et insatisfactions professionnelles dues à l'organisation et aux conditions de travail, ainsi que les impacts que cela avait sur leur vie personnelle, sociale et familiale. À titre d'exemples, mentionnons: les doubles agendas, les longues distances à parcourir, les routes glacées, etc. Par la même méthode, ils ont pris conscience de la dimension collective des effets des conditions de leur travail sur les autres composantes de leur vie. Ils ont aussi échangé sur leurs sentiments respectifs face aux découvertes faites. Ensuite, une analyse du rapport existant entre la perception qu'ils avaient d'eux-mêmes et celle qu'ils croyaient que les autres avaient d'eux leur a révélé une vision plus réaliste de ce qu'ils étaient réellement comme personnes.

Sensibilisation aux contradictions et aux liens travail/famille. D'abord individuellement, puis en groupe, ils ont identifié les contradictions dans les réalités précédemment nommées. Ils les ont liées avec les conditions de travail vécues et ont dégagé le rapport que ces facteurs avaient sur la qualité de leur vie familiale ainsi que les attentes respectives venant du milieu de travail et du milieu familial.

Prise de conscience du contexte structurel des réalités. À chacune des étapes précédentes, ils ont établi des rapports qui les concernaient, entre les contradictions identifiées et les différentes réalités nommées. L'exemple suivant en témoigne : "La majorité d'entre nous consommons de l'alcool lors de certaines rencontres, ou à l'heure du dîner, et cela a l'effet de diminuer le stress au travail. Nous continuons souvent d'en consommer à domicile pour réduire le stress qui se continue au niveau familial. Cela a pour effet d'engendrer des problèmes de communication avec l'épouse et les enfants, de provoquer des périodes d'impuissance sexuelle et on reconsomme pour oublier tout cela ". ${ }^{19}$

Après une période de prise de conscience et afin de voir plus clair dans leur situation, les questions suivantes ont été approfondies : à qui cette situation profite-t-elle? qui en souffre le plus? quel est le prix à payer pour une telle insatisfaction?

Cela a permis d'amorcer un processus de résolution de problème.

Amorce d'un processus de résolution de problème. À partir des réalités identifiées au cours des étapes précédentes, le groupe est rentré dans la dynamique décrite dans les paragraphes suivants.

1) dans une première étape, axée sur le privé et l'individuel: compte tenu de ma personnalité, de mes sentiments et de ma profession, qu'est-ce que je peux faire pour moi, avec les autres participants du groupe? Qu'est-ce que je ne peux pas faire pour moi, avec les autres? 
2) dans une deuxième étape, axée sur le collectif et le communautaire: qu'est-ce qu'on peut faire ensemble pour nous dans notre propre milieu de travail? Qu'est-ce qu'on peut faire ensemble pour d'autres travailleurs comme nous? 20

C'est à cette phase du processus que les participants ont décidé de se donner du pouvoir, mais de façon réaliste, sur leur situation personnelle et collective.

À l'aide d'une grille du processus de résolution de problème ${ }^{21} \mathrm{ils}$ ont vécu individuellement d'abord, puis en groupe, l'élaboration et l'actualisation de solutions concrètes visant l'atteinte d'un nouvel équilibre face au rapport existant entre leurs conditions de travail et les impacts constatés dans les autres dimensions de leur vie.

\section{Limites}

Cette expérience contient les limites du cadre théorique auquel elle se réfère, celles du contexte des réalités directement liées au milieu de travail et au vécu des permanents syndicaux qui y ont participé.

De plus, étant donné le peu de documentation sur ce type d'intervention, surtout si l'on s'en tient à la problématique et aux théories en service social, l'approche structurelle comme approche intégrée, nous n'avons pu nous permettre une analyse aussi approfondie que nous l'aurions souhaitée. Nous avons cependant la prétention d'avoir contribué à l'ouverture et au développement des actions et des théories menant à une intégration de plus en plus structurée des approches utilisées en cours d'expérimentation.

\section{Résultats}

Les membres du groupe ont conçu, individuellement, un projet d'action visant l'augmentation de leur pouvoir pour "changer des choses " afin d'atteindre un équilibre plus satisfaisant face aux différents niveaux de leur vie personnelle et familiale. Dans l'actualisation de ce plan, ils se sont donnés une structure de support en choisissant parmi les autres participants une ou deux personnes qui vérifiaient avec eux le cheminement de leur démarche. Ils ont aussi impliqué les membres de leur famille dans la conceptualisation et l'actualisation du processus entrepris. Cela leur a permis de vivre de nouvelles relations familiales, une nouvelle compréhension de leur dynamique de vie, la création d'une solidarité familiale auparavant absente. Les deux structures de support, famille et collègues de travail, ont été une garantie du sérieux de leur action. 
Dans un deuxième temps, les participants ont élaboré un projet collectif visant à sensibiliser les confrères et consœurs de leur centrale syndicale. Ils se sont approprié le processus et le contenu de l'intervention vécue afin de reproduire cette démarche dans leur milieu de travail immédiat et ainsi tenter d'influencer les autres travailleurs dont ils étaient les représentants et les responsables syndicaux.

À partir du constat qu'ils reproduisaient inconsciemment le malaise vécu et les contradictions (dues au rapport d'insatisfaction entre les conditions de travail et les impacts déjà mentionnés) auprès des travailleurs pour lesquels ils œuvraient à l'amélioration de la qualité de vie au travail, leur motivation à sortir de ce cercle vicieux a soutenu tout le processus d'action pour y remédier. Cela impliquait qu'ils se rendaient responsables des prises de conscience et des décisions à l'effet que l'intervention expérimentée fasse "boule de neige». Ce matériel est donc entre leurs mains et il leur appartient.

\section{Évaluation de l'expérience}

L'évaluation comme processus s'est vécue tout au long des étapes, et les données que nous exposons ici proviennent de la compilation du questionnaire complété par les participants à la fin de l'expérience. ${ }^{22}$

La première partie de ce questionnaire (questions 1 à 42) visait à évaluer le degré de satisfaction des participants en rapport avec les objectifs de l'expérience et les leurs comme personnes, travailleurs, groupe, collectivité de travailleurs. La seconde partie du même questionnaire (questions 43 à 55) voulait surtout mesurer la satisfaction des participants face aux performances professionnelles de l'intervenante.

Qualitativement et globalement, il s'est dégagé un niveau de satisfaction très positif par rapport à l'atteinte des objectifs de l'intervention, ceux des participants ainsi que ceux de l'intervenante.

À titre d'exemple, voici quelques indicateurs de satisfaction identifiés par les participants : tous sont satisfaits du processus vécu ; les principales activités ont favorisé l'atteinte des objectifs de prise de conscience et de gestion du stress, tant sur le plan personnel que professionnel; les activités reliées au processus d'analyse et de solution de problèmes ont été très appréciées ; le climat favorable a permis la mise en commun du vécu émotif; le sentiment d'appartenance au groupe a favorisé le travail personnel et collectif vers l'atteinte des objectifs; la bonne organisation des rencontres et le respect des règles établies ont aussi contribué à l'avancement du travail et même à la croissance des membres ; le matériel utilisé se voulant à la fois méthode et outils peut être réutilisé avec d'autres travailleurs. 
Sans reproduire ici tous les outils utilisés, il s'est dégagé que la prise de conscience des membres face à l'importance du groupe pour générer la synergie nécessaire à l'action, au maintien de cette action pour se l'approprier et pouvoir la transmettre s'est avérée satisfaisante.

La mise sur pied et la réalisation du projet personnel de chacun et au projet collectif pour agir sur leur stress individuel et le stress au travail sont des réalités qu'ils ont vécues. Cela illustre aussi un fort degré d'intégration de la théorie véhiculée dans cet article.

Notons cependant que le volet de reproduction du processus avec d'autres a connu un degré de satisfaction positif mais légèrement inférieur aux projets individuels et à celui du groupe entre eux. Cette action s'inscrivant dans une perspective à plus long terme, nous n'avons pu y vérifier les impacts sur les autres travailleurs. C'est, à notre avis, le point faible du projet. En effet, le départ de l'animatrice pour l'étranger a probablement eu un impact à ce sujet car le suivi s'est vécu par correspondance et son succès n'a pu être validé.

Il ressort que tous se sont dit très satisfaits de leur implication ainsi que de celle de l'animatrice. Dans le cadre du présent article il n'apparaît pas pertinent de tenir compte de cette seconde partie de l'évaluation car elle visait plutôt les habiletés de performance de l'intervenante.

\section{Conclusion}

Par cet article, nous avons voulu illustrer que l'on peut, comme intervenante sociale, utiliser nos connaissances et nos habiletés pour tenter d'intégrer davantage notre action sociale. En effet, nous avions la conviction qu'il était possible de viser à la fois le cheminement personnel et social, en puisant dans les théories du service social, dont celle de l'approche structurelle, et dans celles des approches patronales-syndicales d'intervention en milieu de travail. Il était aussi possible de dégager une équation permettant de développer une complémentarité entre le personnel social et les travailleurs, ainsi qu'une intégration entre les méthodes de service social.

En demeurant fidèle à la logique et au fil conducteur de ce travail, nous ajoutons qu'une telle démarche d'action et d'intégration devrait toujours se vivre en liaison " dialogique » entre les différents partenaires impliqués.

Bien que tout ne puisse être dit dans le cadre et les limites de cet article, il nous semble impossible d'en fermer complètement la boucle car tout repart en spirale vers d'autres possibilités. Cette remarque nous met en garde contre toute étroitesse de vue et permet de donner un 


\section{ANNEXE 1}

\section{Synthèse du processus structurel de l'intervention}

\begin{tabular}{|c|c|c|c|c|}
\hline $\begin{array}{c}-1- \\
\text { Identification } \\
\text { des malaises }\end{array}$ & $\begin{array}{l}-2 \text { - } \\
\text { Quand? } \\
\text { Où? }\end{array}$ & $\begin{array}{c}-3- \\
\text { Identification } \\
\text { du type de stress }\end{array}$ & $\begin{array}{c}-4- \\
\text { Type de } \\
\text { personnalité }\end{array}$ & $\begin{array}{c}-5- \\
\text { Évaluation } \\
\text { de l'état physique }\end{array}$ \\
\hline $\begin{array}{c}-6- \\
\text { Identification des } \\
\text { facteurs stressants } \\
\text { dans une journée }\end{array}$ & \multicolumn{2}{|c|}{$\begin{array}{c}-7- \\
\text { Facteurs personnels } \\
\text { Facteurs contextuels } \\
\text { Facteurs organisationels }\end{array}$} & \multicolumn{2}{|c|}{$\begin{array}{c}-8- \\
\text { Conclusion } \\
\text { de cette étape } \\
\text { du processus }\end{array}$} \\
\hline $\begin{array}{c}-9- \\
\text { Conséquences } \\
\text { des précédentes } \\
\text { étapes }\end{array}$ & \multicolumn{2}{|c|}{$\begin{array}{l}\quad-10- \\
\text { Prise de conscience } \\
\text { et questionnement } \\
\text { sur les impacts }\end{array}$} & \multicolumn{2}{|c|}{$\begin{array}{l}-11- \\
\text { Identification des } \\
\text { sentiments dans la } \\
\text { réalité nommée }\end{array}$} \\
\hline $\begin{array}{c}-12- \\
\text { Identification } \\
\text { des solutions } \\
\text { personnelles... }\end{array}$ & $\begin{array}{l}\quad-13- \\
\ldots . . \text { à court terme } \\
\text { et à long terme }\end{array}$ & $\begin{array}{c}-14- \\
\text { Évaluation } \\
\text { des solutions }\end{array}$ & $\begin{array}{l}\text { - } 15 \text { - } \\
\text { Actualisation } \\
\text { des solutions }\end{array}$ & $\begin{array}{c}-16- \\
\text { Structure } \\
\text { de support }\end{array}$ \\
\hline $\begin{array}{c}-17- \\
\text { Identification } \\
\text { des solutions } \\
\text { collectives... }\end{array}$ & $\begin{array}{l}\quad-18- \\
\ldots \text { à court terme } \\
\text { et à long terme }\end{array}$ & $\begin{array}{c}\text { - } 19 \text { - } \\
\text { Évaluation } \\
\text { des solutions }\end{array}$ & $\begin{array}{l}\quad-20- \\
\text { Actualisation } \\
\text { des solutions }\end{array}$ & $\begin{array}{c}\text { - } 21- \\
\text { Structure } \\
\text { de support }\end{array}$ \\
\hline $\begin{array}{c}-22- \\
\text { Analyse } \\
\text { des résultats } \\
\text { et conclusion }\end{array}$ & $\begin{array}{l}\text { Proj } \\
\text { Pro }\end{array}$ & $\begin{array}{l}\text { iduel } \\
\text { ectif }\end{array}$ & $\begin{array}{l}\quad-24- \\
\text { Évaluation de } \\
\text { l'intervention }\end{array}$ & $\begin{array}{c}-25- \\
\text { Fin }\end{array}$ \\
\hline
\end{tabular}


élan chaque fois qu'un pas se fait vers l'évolution d'une pratique et/ou d'une théorie. C'est aussi en vivant nos doutes que nous donnons un sens à nos questionnements et à nos actions : l'intervention sociale en milieu syndical serait-elle une nouvelle avenue pour le service social?

\section{Notes et références}

* Nous sommes très reconnaissants envers Maurice Moreau, professeur en travail social à l'Université de Montréal, René Auclair et Jocelyn Lindsay, professeurs en travail social à l'Université Laval, Rosario Lopez-Tremblay, travailleuse sociale au Carrefour Ubald-Villeneuve à Sainte-Foy, Ghislain Ross, travailleur syndiqué, pour leur précieuse collaboration dans l'élaboration de cet article. Un merci spécial s'adresse aux permanents syndicaux concernés, car sans leur participation au projet, cet article n'aurait pu être écrit. Merci également à Adrien Lacroix, directeur général de La Clairière de Québec et du Carrefour Ubald-Villeneuve ainsi qu'à mes collègues de travail pour leur support moral, le service de dactylographie, leur tolérance de mes absences pendant le travail de rédaction.

1 Considérant que la majorité des intervenants sociaux sont des femmes, nous utilisons dans le présent article le genre féminin. Le lecteur peut donc intervertir les deux genres selon la lecture qu'il lui plaît de faire.

2 Nous utilisons ici le genre masculin car tous les conseillers syndicaux rencontrés étaient des hommes. Voir aussi l'article de Maurice Moreau et Michelle Desrosiers, "Le partenaire invisible ; les conditions de travail dans l'évaluation et l'intervention sociale ", Service Social, vol. 35, no 3, $1986: 375-385$.

3 Home, Alice et Darveau-Fournier, Lise, La pratique du service social des groupes au Québec, Rapport final de l'étude "Utilisation des groupes en service social dans la région 03 ", Laboratoire de recherche, École de service social, Université Laval, Québec, 1981.

Home, Alice et Darveau-Fournier, Lise, "La spécificité du service social des groupes", Service Social, vol. 29, nos 1-2, 1980 : 16-30.

4 Desmarais, Danielle, "Stress, santé mentale et syndicalisme: l'approche de I'Institute for Labor and Mental Health ", Revue internationale d'action communautaire, no 7/47, 1982 : 179-186.

Marshall, Judy et CoOper, Cary L., Coping with Stress at Work, London, Gower Publishing Company Limited, 1981.

LERNER, Michael P., Stress at the Work Place, The Approach of the Institute for Labor and Mental Health, Oakland (Cal.), 1979, document miméographié.

MASLACH, Christina, "Job Burnout-How People Cope ", Public Welfare, vol. 36, no 2, 1978: 56-58.

5 Marshall, Judy et Cooper, Cary, op. cit. 
6 Pines, Ayala ; Aronson, Elliot et Kafry, Ditsa, Se vider dans la vie et le travail, Montréal, Éditions du jour, 1982.

7 LERNER, Michael, op. cit.

8 Moreau, Maurice, "L'approche structurelle familiale en service social: le résultat d'un itinéraire critique ", Revue internationale d'action communautaire, no 7/47, 1982: 159-171.

Moreau, Maurice et Desrosiers, Michelle, op. cit.

9 Moreau, Maurice, op. cit., p. 159.

10 Idem.

11 Idem.

12 Idem.

13 Darveau-Fournier, Lise et Home, Alice, "Les groupes de service social à Québec : recherche des rapports entre théorie et pratique", Service Social, vol. 32, nos 1-2, $1983:$ 129-155.

14 Moreau, Maurice et Desrosiers, Michelle, op. cit.

15 BOUCHER, Francine et BINETTE, André, Bien vivre le stress, Montréal, Éditions de Mortagne, 1981.

BERGERON, Jean-Louis ; CÔtÉ-LÉGer, Nicole ; JACQUes, Jocelyn et BélANGER, Laurent, Les aspects humains de l'organisation, Chicoutimi, Gaëtan Morin, 1979.

Centrale des Syndicats démocratiques, La base des nouveaux syndicalistes québécois, des milieux de travail à réinventer ensemble, Cahier spécial, Montréal, C.S.D., 1982.

DesmaraIs, Danielle, op. cit.

LERNER, Michael P., op. cit.

Norfolk, Donald, Le stress dans le monde des affaires, Montréal, Éditions du jour, 1980.

PAGÈs, Max et DESCENDRE, Daniel, «Recherche sur les phénomènes de pouvoir dans les grandes organisations industrielles ", Sociologie et Sociétés, vol. 9, no 2, 1977: 122-147.

16 BouCher, Francine et BINetTe, André, op. cit.

BRAMHALL, Martha et EzelL, Susan, "How burned out are you ? ", Public Welfare, vol. 39 , no 1,1981 : 23-28. Traduction du questionnaire de la page 28 par Jocelyn Lindsay.

17 BouCher, Francine et BinetTe, André, op. cit.

18 Bramhall, Martha et Ezell, Susan, op. cit.

19 Témoignage recueilli par l'auteure lors de l'intervention.

20 Middleman, Ruth R. et Goldberg, Gale, Social Service Delivery, a Structural Approach to Social Work, New York, Columbia University Press, 1974.

21 BouCher, Francine et BINETTE, André, op. cit.

22 Le questionnaire de 55 questions regroupait les trois niveaux suivants : personnel, groupe et collectivité. Il serait trop long de le reproduire dans le cadre du présent article. Pour plus de précision, le lecteur peut communiquer avec l'auteure. 